$$
\text { الأهمية النسبية لبعض المعايير المكانية المساهمة تحديد مسار الطريق الخارجية }
$$

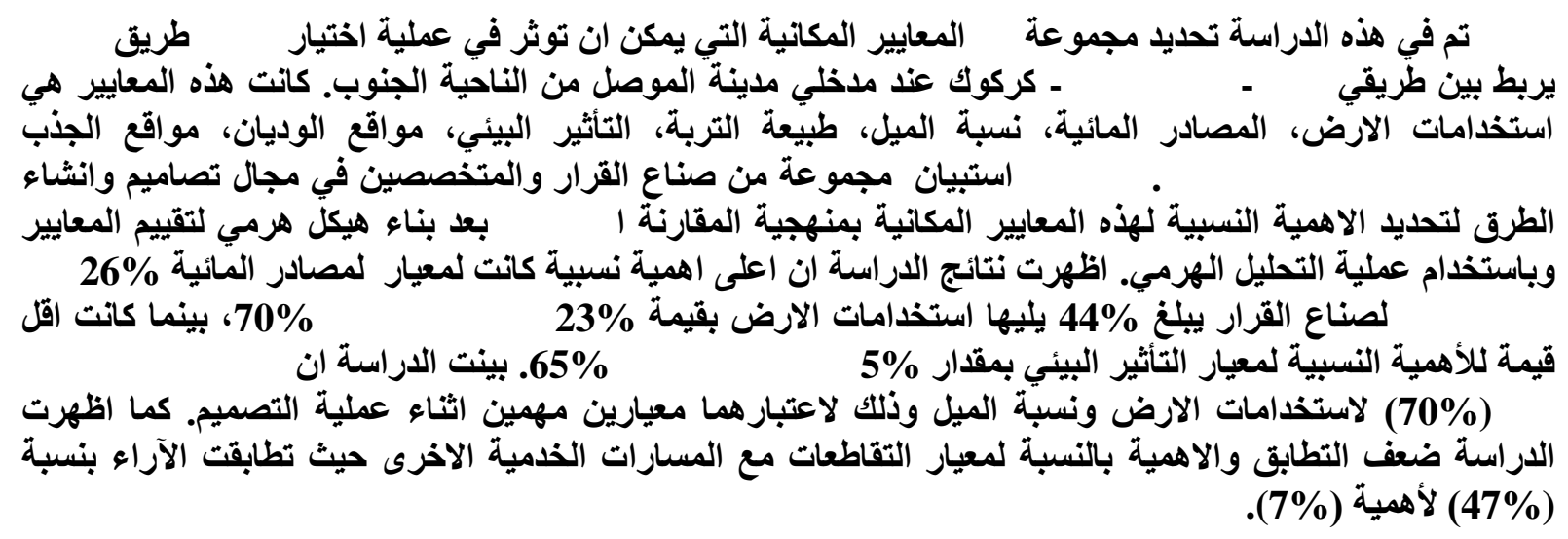

\title{
The Relative Importance of some Spatial Criteria contribute the Route Location for rural highway
}

\author{
Dr. Ayman Abd Alhadi
}

Mohammed Ghanim Jamel

University of Mosul / College of Engineering / Civil Engineering

\begin{abstract}
This study was conform determine the set of criteria spatial affect the process of selecting the site route location of a road linking between Mosul - Baghdad and MosulKirkuk highway at the southerner entrances of Mosul city. These criteria were a land use, water resources, slope, geology, environmental impact, hydrology, control area and linear engineering structure. Questionnaire from was distributed to a group of decisionmakers and specialists in the field of design and road construction to determine the relative importance of these criteria spatial using Pairwise comparison was conducted after building a hierarchical structure to evaluate the standards and using the analytic hierarchy process (AHP). The study results showed that the highest relative importance criteria was the water sources $26 \%$ when the ratio of the consensus of $44 \%$, followed by the uses of the land value of $23 \%$ when the ratio of the consensus $70 \%$, while value of the relative importance was the standard environmental impact by $5 \%$ and the rate at a consensus of $65 \%$. The high consensus at $(70 \%)$ of the uses of the land and slope of the grounds are important criteria during the design process. The study also showed less consensus and important standard for linear engineering structure where tracks matched by consensus $(47 \%)$ of the importance of $(7 \%)$.
\end{abstract}

$6-6-2015:$

$3-5-2014:$ 
هناك العديد من المعايير التي توثر على تحديد مسار الطريق منها الطبيعية كنوع التربة التي يمر عليها الطيا فالطريق

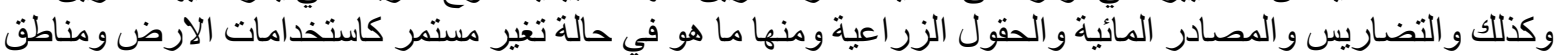

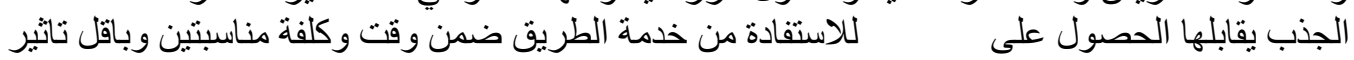

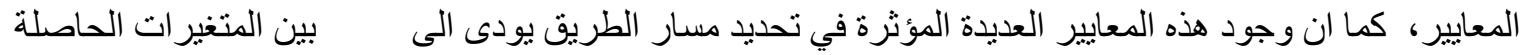

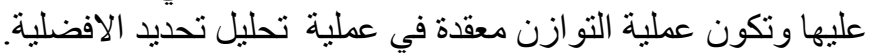

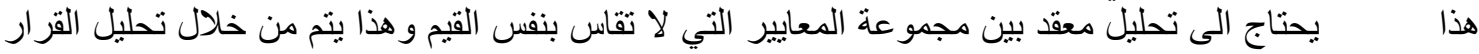
المتعدد المعايير (Multi Criteria Decision Analysis _MCDA) التي ترتبط مع بعضها بموقع التحليل مكونة مجموعة من المعايير التي تقاس نوعا او كما.

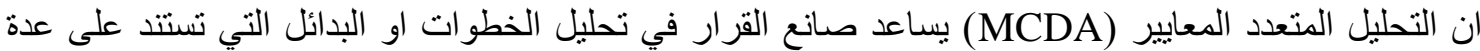

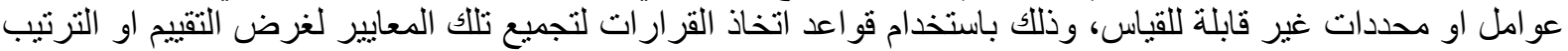
على الرغم من ان معيار القرار لا يمكن ان يكون الحد الاقصى في جميع خيار ات التيدائل التئل

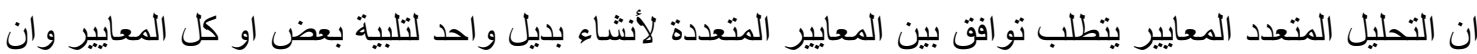

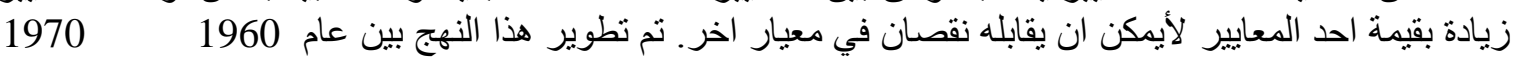

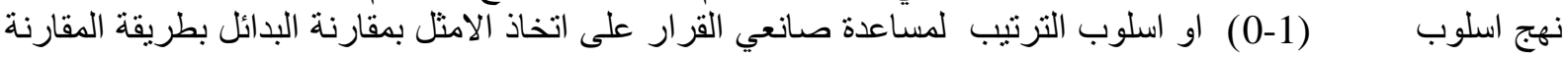

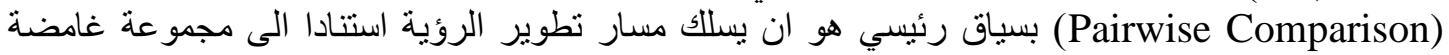

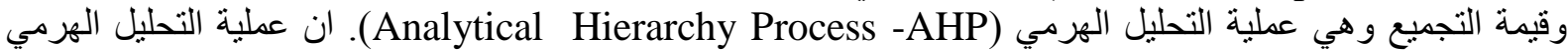

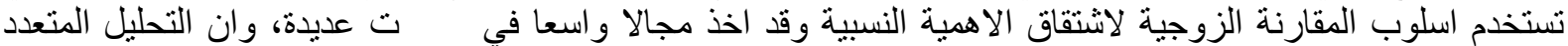
المعايير له القدرة على النظر بوقت واحد الى كل المعايير الكمية والنوعية وبهذا يمكن ان تمثل بمقياس ترتيبي ومستمر [1].

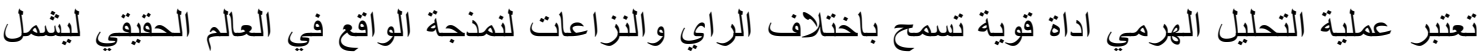

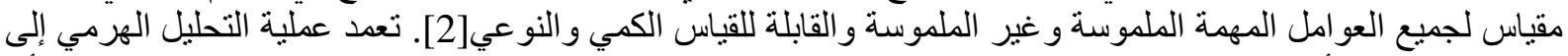

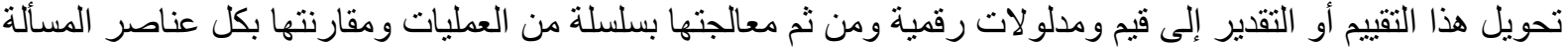

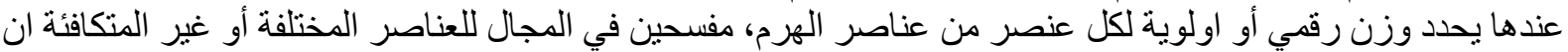

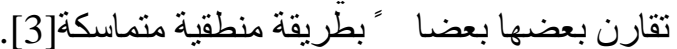
ان عملية التحليل الهرمي

1 التحليل (Decomposition): يتطلب مبدا التحليل بان تتحلل مشكلة القرار بتسلسل هرمي والذي يـ العناصر الاساسية للمشكلة (العبلة

(Comparative Judgment) 2

في مستويات التحليل الهرمي .

3

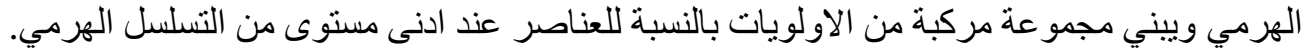

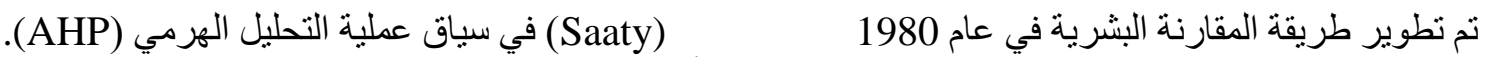

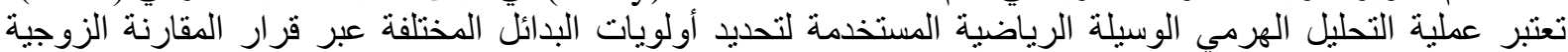

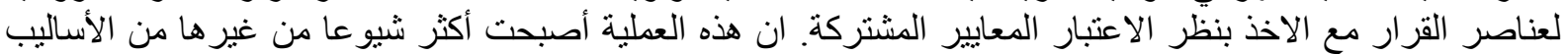

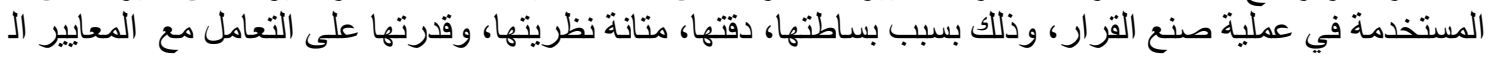
غير المادية[2].

وتكون خطو ات عملية التحليل الهرمي ضمن المر احل التالية [3]

1 2 البشرية ، كل عنصر بمستوى مع مجمو عة العناص العناصر بمستو ياتها المختلفة. 
4 الاقتخدام الاولويات التي نم الحصول عليها من المقارنات ، تحديد الاولويات للمستوى الاعلى وثم يليها المستوى

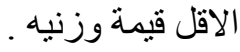

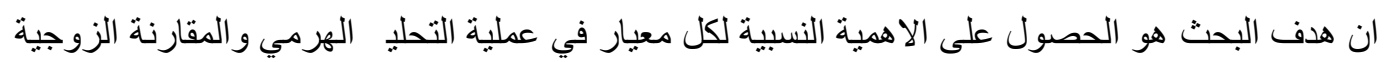
بين المعايير ضمن مصفوفة التعبير التي تمثل مدى التجانس بين القية (Consistency Ratio)

\section{منهاج \\ المعايير الداخلة في عملية التحليل الهرمي:}

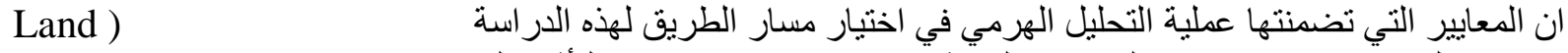

use ميل الارض (Slope) المصادر المائية (Water Resource) التأثير البيئي (Environmental Impact)

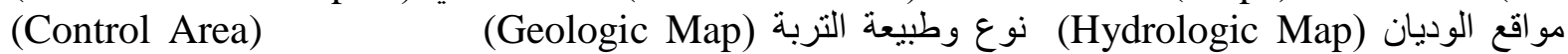
مسار العوارض الخطية (Linear Engineering Structure).

مدينة الموصل لتسهيل

حركة المركبات من خارج حدود المقبط بين طريقي بغداد-

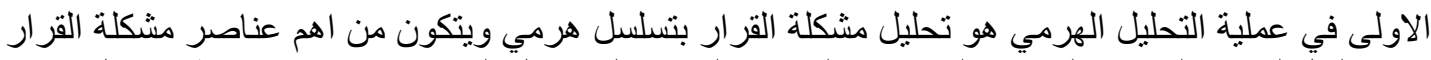

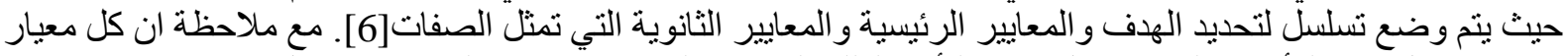

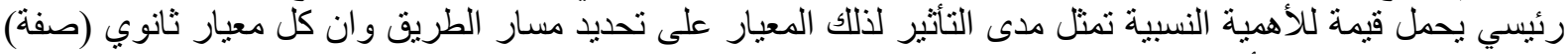

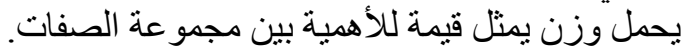

\section{تقييم اوزان المعايير:}

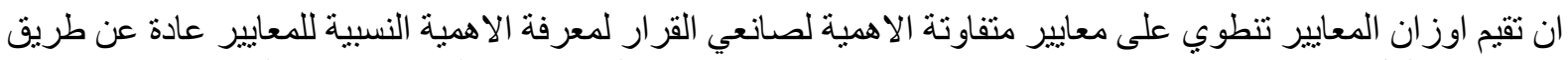

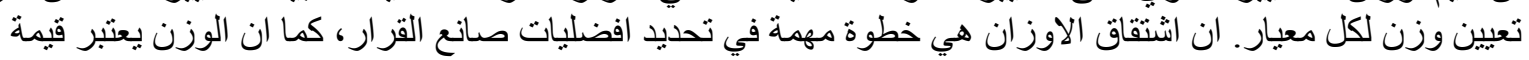

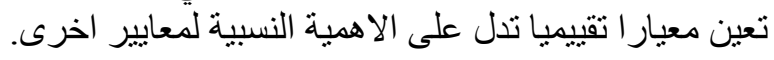

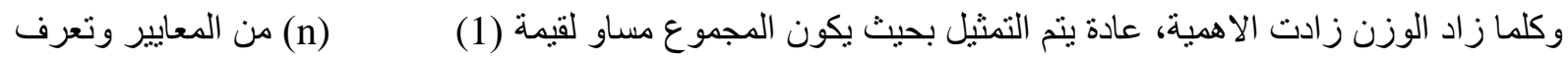

$W=(w 1, w 2, \ldots w j, \ldots w n)$

$\sum W j=1$

ويمكن اتباع مقياس المقارنة الزوجية كأحد طرق تحديد هذ

تحديد الاهمية النسبية للمعاييز:

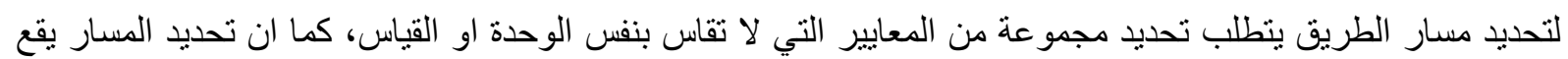

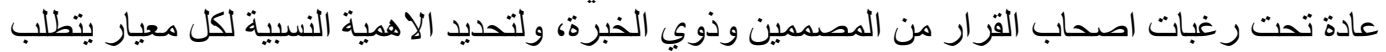

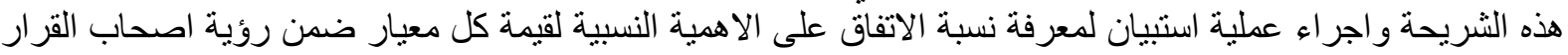

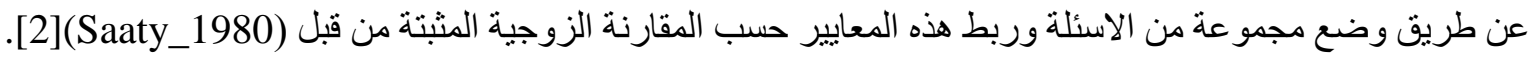

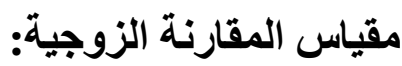

يعتبر مقياس المقارنة الزوجية واحدة من الخطوات الأكثر أهمية في العديد من وسائل صنع القرار وهو التهو تقدير دقيق للبيانات

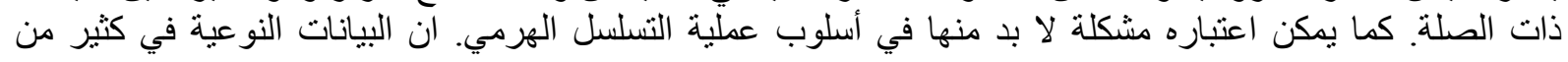


الأحيان لا يمكن أن تكون معروفة من حيث القيم المطلقة، لذا من الصعب جدا إن لم يكن مستحيلا تحديدها بشكل صحيح.

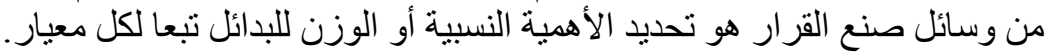

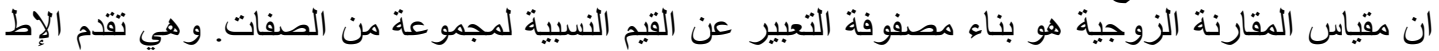

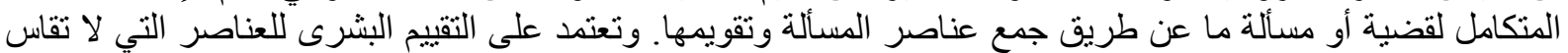

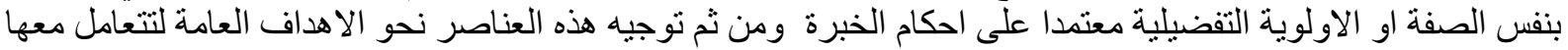

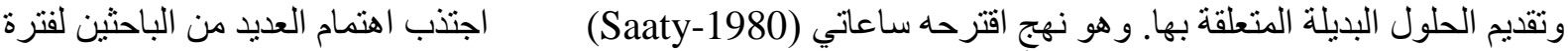

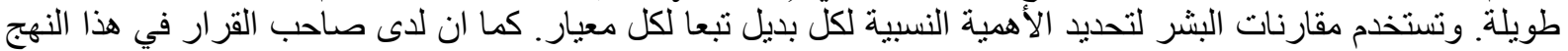

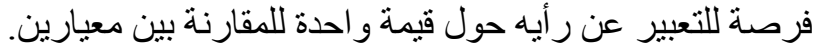

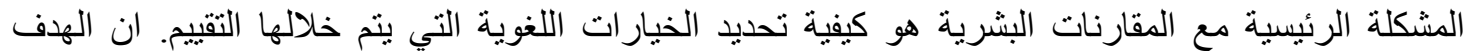

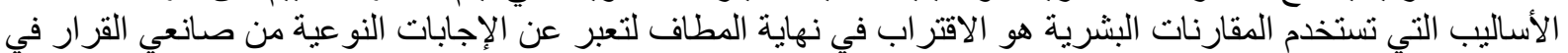

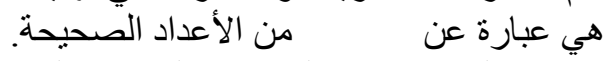

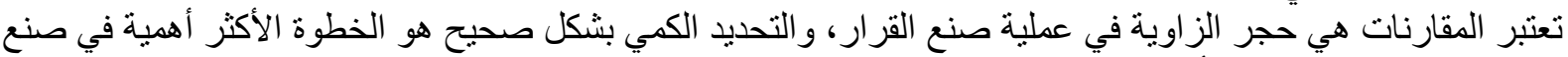

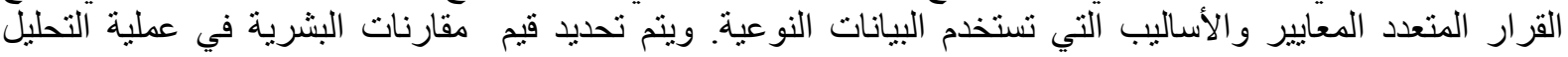

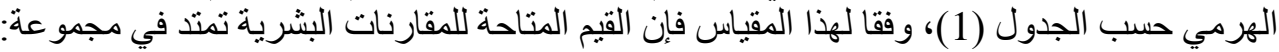

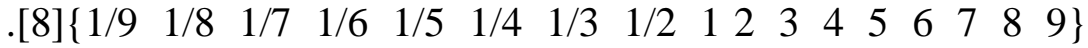

[2](Saaty-1980(1) قيم الاهمية في تحديد مقياس المقارنة الزوجية المقترحة من قبل

\begin{tabular}{|c||c||l|}
\hline $\begin{array}{c}\text { Intensity of } \\
\text { Importance }\end{array}$ & Definition & \multicolumn{1}{c|}{ Explanation } \\
\hline \hline 1 & Equal importance & $\begin{array}{l}\text { Two activities contribute equally to the } \\
\text { objective }\end{array}$ \\
\hline 3 & $\begin{array}{c}\text { Weak importance of } \\
\text { one over another }\end{array}$ & $\begin{array}{l}\text { Experience and judgment slightly favor } \\
\text { one activity over another }\end{array}$ \\
\hline 5 & $\begin{array}{c}\text { Essential or strong } \\
\text { importance }\end{array}$ & $\begin{array}{l}\text { Experience and judgment strongly favor } \\
\text { one activity over another }\end{array}$ \\
\hline 7 & $\begin{array}{c}\text { Demonstrated } \\
\text { importance }\end{array}$ & $\begin{array}{l}\text { An activity is strongly favored and its } \\
\text { dominance demonstrated in practice }\end{array}$ \\
\hline $2,4,6.8$ & $\begin{array}{c}\text { Absolute importance } \\
\text { Intermediate values } \\
\text { between the two } \\
\text { adjacent judgments }\end{array}$ & $\begin{array}{l}\text { The evidence favoring one activity over } \\
\text { another is of the highest possible order of } \\
\text { affirmation }\end{array}$ \\
\hline
\end{tabular}

\section{حساب الاهمية النسبية للمعايير}

بعد القيام بعملية تحديد المعايير و التي تؤثر بتحديد موقع المسار و اعطاء وصف عام لكل منها، يجب تحديد وزن لكل منها

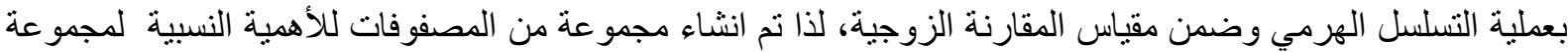

تدخل في هذه العملية مجموعة المعايير، تكون المقارنة بين نفس المعيار بقيمة اهمية متساوية اي تمثل قيمة (1). بينما

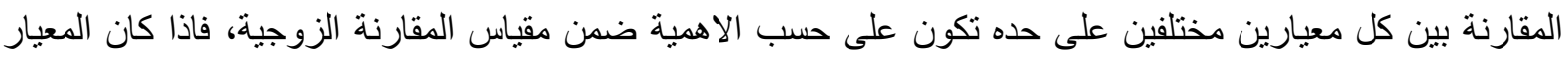

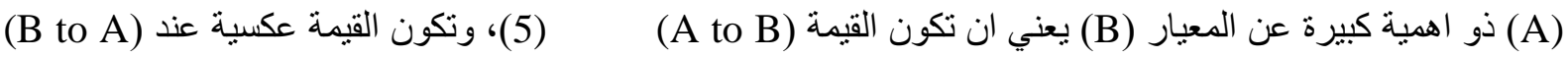

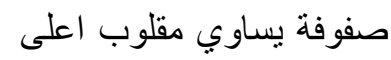

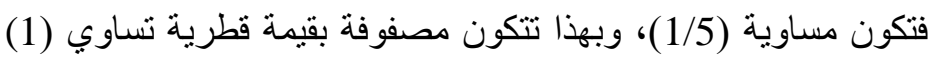


: الأهمية النسبية لبعض المعايير المكانية المساهمة تحديد مسار الطريق الخارجية

.[9]

$$
C i j=>_{i=1}^{n} C i j
$$

الخطوة الثانية : تقسم كل قيمة بالعمود على مجموع العمود من المصفوفة [9]

$$
X i j=\frac{C i j}{\sum_{i=1}^{n} C i j}
$$

(n)

$$
\text { يتم احتساب الاوزان للمعايير بعد تكوين }
$$




$$
\begin{aligned}
& C v 11=\frac{1}{w 11}[C 11 W 11+C 12 W 21+C 13 W 31] \\
& C v 21=\frac{1}{w 21}[C 21 W 11+C 22 W 21+C 23 W 31] \\
& C v 31=\frac{1}{w 31}[C 31 W 11+C 32 W 21+C 33 W 31]
\end{aligned}
$$

$$
\text { لحساب قيمة ( ) وقيمة (CI) وقيمة الاتساق (CR) [9]. }
$$

$$
=\frac{\sum_{i=1}^{n} C v}{n}
$$

$$
C I=\frac{\lambda-n}{n-1}
$$

$$
C R=\frac{C I}{R I}
$$

\section{عملية استبيان اصحاب القرار:}

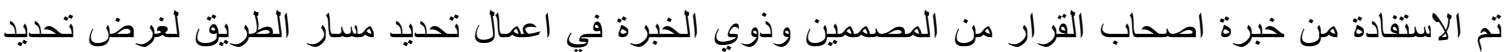

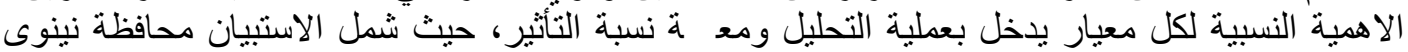

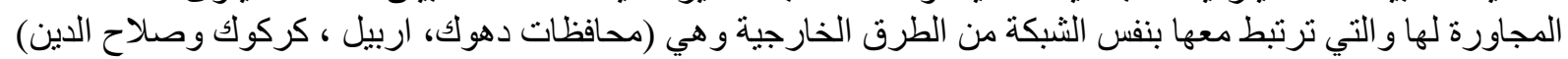

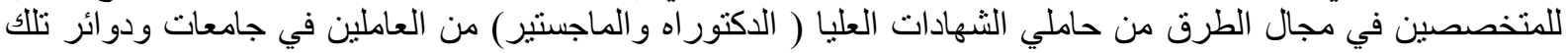

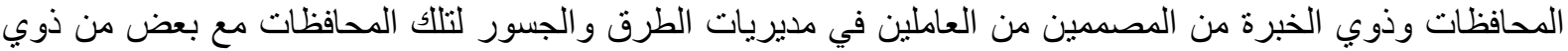
الخبرة المتخصصين و العاملين بالقطاع الخاص، شكل (1) يوضح استمارة الاستبيان المعتمدة في عملية الاستبيان.

(Consistency Ratio -CR) تحليل بيانات اراء صناع القزار:

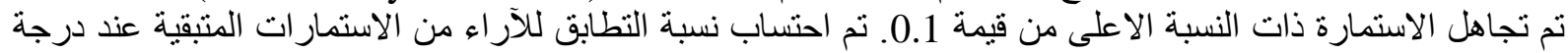
لمعرفة نسبة التطابق (درجة الاجماع)، حيث تعتبر درجة الاجماع كمقرر رئيسي الإني (Confidence Interval-99\%) لطبيعة عملية الاختيار [3]، عن طريق اهمال النتائج الغير منطقية و المخالفة للحد الاعلى الألى

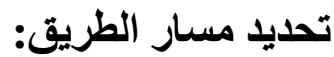
يتم توحيد المعايير المكانية بمجموعة رقيلة رقمية مشتركة ومجتمعه عن طريق معدل الاوزان، وذللك بعد تعين صناع القرار

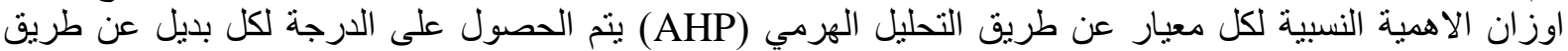

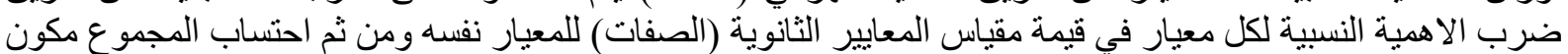
بذلك نتيجة اجمالية.

يمكن تنفيذ هذا الاسلوب باستخدام نظم المعلومات الجغر افية (GIS) بواسطة قدرة التركيب (Overlay Capabilities)

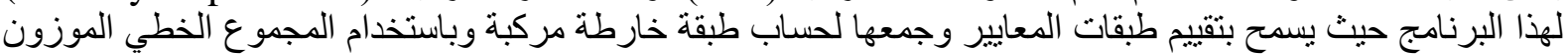
يتم الجمع بتطبيق الاوز ان للحصول على خارطة الملائمة (WLC) (7) (2uitability Map). 


\section{: الأهمية النسبية لبعض المعايير المكانية المساهمة تحديد مسار الطريق الخارجية}

\section{الأهمية النسبية للمعايير المستخدمة في اختياز المسار الأفضل:}

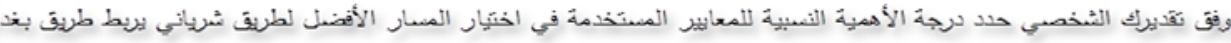

كركويك عند مداخل مدينة الموصن من نوع ( 2 way-4lane )

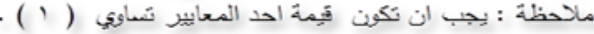

Land use أنخدامن الارضن

Land use استخدامت الارضن

Land use استخدامت الارض انت

Land use استخدامك الارضن

Land use استخداملت الارن

Land use ابتخدامك الارضن

Land use استخدامك الارض

Water Source المحسادر المبأية

Water Source المصادر المئية

Water Source المصادر المسئية

Water Source المصادر الملئية

Water Source المسيادر المسئية

Water Source المصادر المبأية

Slope الميل المبل المبل Slope
Slope
Slope
Slope

نوع الثربة Geological نوع التربة Geological نوع الثربة Geological نوع التربة Geological نوع التربة
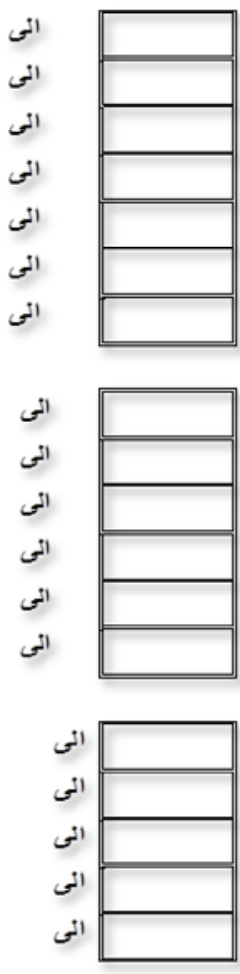

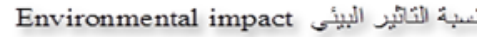

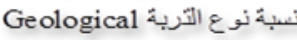

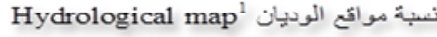

Control point ${ }^{2}$ نبية نقلط الجنب

انسبة منسار العوارض الخدمية

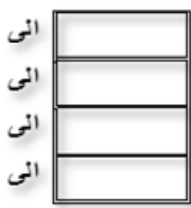

Environmental impact نببة الثأثير البيئي

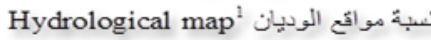

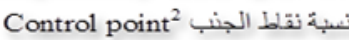

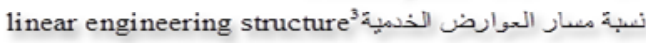

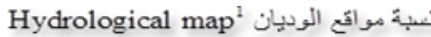

Control point ${ }^{2}$ نببة نقلط الجنب

انسبة مسار العوارض الخدمية3

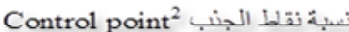
انسبة مندار العوارض الخدمية 


\section{-}

rar


: الأهمية النسبية لبعض المعايير المكانية المساهمة تحديد مسار الطريق الخارجية

(5)

\begin{tabular}{|c|c|c|}
\hline Criteria & Step 1 & Step 2 \\
\hline Land use(LU) & $(0.2259 * 1+0.2643 * 0.5+0.0904 * 4+0.1036 * 2+0.0424 * 6+0.0849 * 3+0.119 * 2+0.0692 * 5)=2.021$ & $2.021 / 0.2259=8.946$ \\
\hline $\begin{array}{c}\text { Water } \\
\text { Source(WS) }\end{array}$ & $\left(0.2259 * 2+0.2643^{*} 1+0.0904^{*} 5+0.1036 * 4+0.0424 * 4+0.0849 * 4+0.119^{*} 2+0.0692 * 3\right)=2.368$ & $2.368 / 0.2643=8.961$ \\
\hline Slope(SL) & $\left(0.2259^{*} 0.25+0.2643 * 0.2+0.0904 * 1+0.1036^{*} 1+0.0424 * 3+0.0849 * 2+0.119 * 0.5+0.0692 * 2\right)=0.7987$ & $0.7987 / 0.0904=8.828$ \\
\hline Geological(GL) & $\left(0.2259^{*} 0.5+0.2643 * 0.25+0.0904 * 1+0.1036^{*} 1+0.0424 * 2+0.0849 * 2+0.119 * 2+0.0692 * 0.5\right)=0.9006$ & $0.9006 / 0.1036=8.686$ \\
\hline $\begin{array}{l}\text { Environmental } \\
\text { Impact(EI) }\end{array}$ & $\begin{array}{l}(0.2259 * 0.16+0.2643 * 0.25+0.0904 * 0.33+0.1036 * 0.5+0.0424 * 1+0.0849 * 0.5+0.119 * 0.5+0.0692 * 0.5) \\
=0.3647\end{array}$ & $0.3647 / 0.0424=8.584$ \\
\hline $\begin{array}{l}\text { Hydrological } \\
\text { Map (HL) }\end{array}$ & $\begin{array}{l}\left(0.2259^{*} 0.33+0.2643 * 0.5+0.0904 * 0.5+0.1036 * 0.5+0.0424 * 2+0.0849 * 1+0.119 * 0.5+0.0692 * 3\right) \\
=0.7415\end{array}$ & $0.7415 / 0.0849=8.733$ \\
\hline $\begin{array}{c}\text { Control } \\
\text { Area(CA) }\end{array}$ & $\begin{array}{l}(0.2259 * 0.5+0.2643 * 0.5+0.0904 * 2+0.1036 * 0.5+0.0424 * 2+0.0849 * 2+0.119 * 1+0.0692 * 3)= \\
1.0593\end{array}$ & $1.0593 / 0.119=8.900$ \\
\hline $\begin{array}{c}\text { Linear } \\
\text { Engineering } \\
\text { Structure(LES) }\end{array}$ & $\begin{array}{l}\left(0.2259^{*} 0.2+0.2643^{*} 0.33+0.0904 * 0.5+0.1036 * 2+0.0424 * 2+0.0849 * 0.33+0.119 * 0.33+0.0692 * 1\right) \\
=0.6080\end{array}$ & $0.6080 / 0.0692=8.785$ \\
\hline
\end{tabular}

نتائج عملية الاستبيان:

تم احتساب الاهمية النسبية للمعايير من استمارات عملية الاستبيان واحتساب قيمة نسبة الاتساق لتحديد وتقيم صحة الإنة

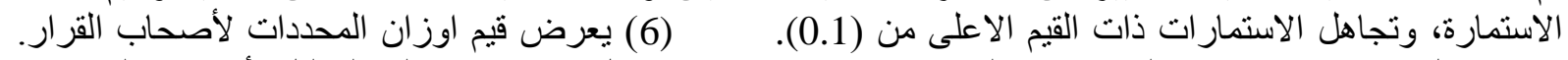

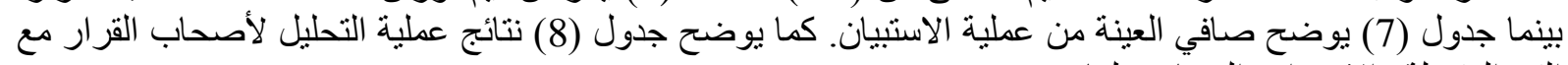

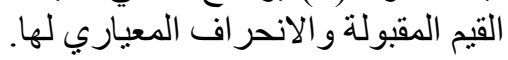


Al-Rafidain Engineering Vol. $23 \quad$ No. $4 \quad$ October 2015

(6) قيم اوز ان المحددات لأصحاب القرار

\begin{tabular}{|c|c|c|c|c|c|c|c|c|c|}
\hline Criteria & LU & WS & SL & GL & EI & $\mathrm{HL}$ & $\mathrm{CA}$ & L.E.S. & C.R \\
\hline Valuerl & 21.91 & 30.70 & 3.95 & 4.42 & 17.72 & 9.25 & 7.36 & 4.71 & 0.078 \\
\hline Valuer $2^{*}$ & 35.77 & 6.26 & 3.83 & 9.51 & 4.01 & 5.08 & 20.23 & 15.30 & 0.283 \\
\hline Valuer3 & 22.83 & 21.79 & 12.70 & 12.19 & 4.04 & 8.33 & 8.45 & 9.66 & 0.048 \\
\hline Valuer4 & 24.83 & 21.11 & 8.69 & 12.74 & 5.17 & 10.83 & 8.36 & 8.27 & 0.071 \\
\hline Valuer5 & 26.22 & 26.22 & 8.10 & 11.00 & 3.30 & 10.61 & 8.63 & 5.94 & 0.093 \\
\hline Value 6 & 24.56 & 26.44 & 10.59 & 11.67 & 3.51 & 4.80 & 13.44 & 4.99 & 0.073 \\
\hline Valuer7 & 26.25 & 27.90 & 7.35 & 9.73 & 2.91 & 4.60 & 9.88 & 11.38 & 0.096 \\
\hline Valuer8 & 22.01 & 26.02 & 11.72 & 13.98 & 2.80 & 9.96 & 9.75 & 3.77 & 0.066 \\
\hline Valuer9 & 3.44 & 26.74 & 13.94 & 9.60 & 2.29 & 16.84 & 12.96 & 14.19 & 0.070 \\
\hline Value 10 & 22.59 & 26.43 & 9.05 & 10.37 & 4.25 & 8.49 & 11.90 & 6.92 & 0.081 \\
\hline Valuer $11^{*}$ & 25.00 & 10.51 & 12.02 & 7.72 & 10.66 & 10.99 & 11.93 & 11.17 & 0.328 \\
\hline Valuer 12 & 16.34 & 28.12 & 32.86 & 4.86 & 3.13 & 5.18 & 5.27 & 4.23 & 0.089 \\
\hline Valuer $13^{*}$ & 13.83 & 8.79 & 10.85 & 13.58 & 10.07 & 16.00 & 14.79 & 12.09 & 1.028 \\
\hline Valuer14 & 25.95 & 25.95 & 10.49 & 9.02 & 6.08 & 7.67 & 9.00 & 5.84 & 0.099 \\
\hline Value 15 & 24.76 & 29.69 & 10.30 & 9.40 & 2.94 & 7.26 & 10.65 & 5.01 & 0.060 \\
\hline Valuer 16 & 22.99 & 5.88 & 2.85 & 4.77 & 22.28 & 5.17 & 24.71 & 11.35 & 0.073 \\
\hline Valuer 17 & 21.29 & 28.70 & 8.80 & 10.09 & 3.75 & 8.37 & 11.71 & 7.30 & 0.091 \\
\hline Valuer 18 & 21.29 & 28.70 & 8.80 & 10.09 & 3.75 & 8.37 & 11.71 & 7.30 & 0.091 \\
\hline Valuer 19 & 24.80 & 26.06 & 10.11 & 9.38 & 3.76 & 8.58 & 12.14 & 5.17 & 0.066 \\
\hline Valuer 20 & 26.15 & 27.58 & 8.37 & 12.14 & 4.09 & 5.65 & 10.09 & 5.93 & 0.029 \\
\hline Valuer $21^{*}$ & 11.39 & 7.86 & 8.61 & 5.87 & 16.08 & 14.02 & 21.47 & 14.69 & 1.343 \\
\hline Valuer 22 & 5.42 & 2.54 & 30.15 & 30.15 & 2.58 & 18.04 & 5.70 & 5.42 & 0.026 \\
\hline Valuer 23 & 40.23 & 13.38 & 6.57 & 5.93 & 6.09 & 7.81 & 12.66 & 7.33 & 0.074 \\
\hline Valuer24 & 24.95 & 37.26 & 4.54 & 7.03 & 5.91 & 7.85 & 6.70 & 5.75 & 0.080 \\
\hline Valuer25 & 24.41 & 22.48 & 8.71 & 12.36 & 6.83 & 5.05 & 10.88 & 9.27 & 0.067 \\
\hline Valuer26 & 24.00 & 19.76 & 9.10 & 14.61 & 7.40 & 6.51 & 11.30 & 7.32 & 0.072 \\
\hline Valuer 27 & 23.51 & 21.59 & 8.18 & 15.19 & 6.23 & 7.04 & 12.47 & 5.81 & 0.040 \\
\hline Valuer 28 & 24.97 & 19.91 & 9.40 & 18.00 & 8.59 & 3.47 & 10.44 & 5.21 & 0.060 \\
\hline Valuer 29 & 21.44 & 28.77 & 8.87 & 10.06 & 3.75 & 8.48 & 11.06 & 7.56 & 0.086 \\
\hline Valuer 30 & 21.64 & 28.89 & 8.94 & 10.06 & 3.76 & 7.82 & 11.13 & 7.76 & 0.077 \\
\hline Valuer 31 & 22.67 & 37.26 & 4.03 & 10.49 & 6.11 & 7.53 & 5.93 & 5.99 & 0.091 \\
\hline Valuer32 & 21.27 & 28.69 & 8.75 & 10.84 & 3.72 & 8.23 & 10.93 & 7.57 & 0.095 \\
\hline \begin{tabular}{|l|} 
Valuer 33 \\
\end{tabular} & 26.78 & 26.78 & 13.85 & 5.08 & 4.03 & 7.21 & 12.93 & 3.34 & 0.012 \\
\hline Valuer 34 & 21.96 & 29.64 & 9.61 & 10.54 & 4.72 & 8.36 & 10.89 & 4.29 & 0.099 \\
\hline Valuer35 & 26.43 & 25.24 & 11.18 & 9.03 & 6.34 & 6.60 & 12.02 & 3.17 & 0.084 \\
\hline Valuer36 & 23.83 & 32.18 & 10.15 & 7.17 & 4.73 & 7.17 & 10.26 & 4.50 & 0.047 \\
\hline Valuer 37 & 23.60 & 25.58 & 9.64 & 9.57 & 4.43 & 8.42 & 11.63 & 7.12 & 0.091 \\
\hline Valuer38 & 23.36 & 24.71 & 8.95 & 10.67 & 4.41 & 8.46 & 11.79 & 7.63 & 0.093 \\
\hline
\end{tabular}

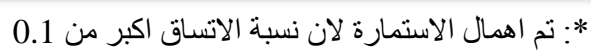

(7) صافي العينة من اصحاب القزار

\begin{tabular}{|c|c|}
\hline 38 & عدد المقيمين من اصحاب القرار \\
\hline 4 & عدد المقيمين خار ج الحدود لقيمة (CR>0.1) \\
\hline 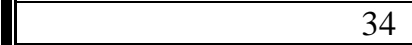 & صافي العينة \\
\hline
\end{tabular}


: الأهمية النسبية لبعض المعايير المكانية المساهمة تحديد مسار الطريق الخارجية

(8) نتائج عملية التحليل لأصحاب القرار مع القيم المقبولة والانحر اف المعياري

\begin{tabular}{|c|c|c|c|c|c|c|c|c|c|}
\hline Criteria & Landuse & Water Source & Slope & Geology & Environmental & Hydrology & Control Area & L.E.S. & Sum Weight \\
\hline 1 & 21.91 & 26.22 & 12.70 & 12.19 & 4.04 & 9.25 & 9.88 & 5.94 & \\
\hline 2 & 22.83 & 26.44 & 8.69 & 12.74 & 5.17 & 8.33 & 9.75 & 6.92 & \\
\hline 3 & 24.83 & 27.90 & 8.10 & 11.00 & 4.25 & 8.49 & 11.90 & 5.84 & \\
\hline 4 & 24.56 & 26.02 & 10.59 & 11.67 & 6.08 & 7.67 & 10.65 & 7.30 & \\
\hline 5 & 22.01 & 26.74 & 11.72 & 9.73 & 3.75 & 7.26 & 11.71 & 7.30 & \\
\hline 6 & 22.59 & 26.43 & 9.05 & 9.60 & 3.75 & 8.37 & 11.71 & 5.93 & \\
\hline 7 & 24.76 & 28.12 & 10.49 & 10.37 & 3.76 & 8.37 & 12.14 & 7.33 & \\
\hline 8 & 22.99 & 25.95 & 10.30 & 9.02 & 4.09 & 8.58 & 10.09 & 5.75 & \\
\hline 9 & 21.29 & 26.06 & 8.80 & 9.40 & 6.09 & 7.81 & 10.88 & 7.32 & \\
\hline 10 & 21.29 & 27.58 & 8.80 & 10.09 & 5.91 & 7.85 & 11.30 & 5.81 & \\
\hline 11 & 24.80 & 22.48 & 10.11 & 10.09 & 6.83 & 7.04 & 10.44 & 7.56 & \\
\hline 12 & 24.95 & 26.78 & 8.37 & 9.38 & 6.23 & 8.48 & 11.06 & 7.76 & \\
\hline 13 & $24.4 !$ & 25.24 & 8.71 & 12.14 & 3.75 & 7.82 & 11.13 & 5.99 & \\
\hline 14 & 24.00 & 25.58 & 9.10 & 12.36 & 3.76 & 7.53 & 10.93 & 7.57 & \\
\hline 15 & 23.51 & 24.71 & 8.18 & 10.06 & 6.11 & 8.23 & 10.89 & 7.12 & \\
\hline 16 & 24.97 &.. & 9.40 & 10.06 & 3.72 & 7.21 & 12.02 & 7.63 & \\
\hline 17 & 21.44 & .. & 8.87 & 10.49 & 4.03 & 8.36 & 10.26 & 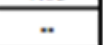 & \\
\hline 18 & 21.64 & .. & 8.94 & 10.84 & 4.72 & 7.17 & 11.63 & .. & \\
\hline 19 & 22.67 & .. & 8.75 & 10.54 & 6.34 & 8.42 & 11.79 &. & \\
\hline 20 & 21.27 &.. & 9.61 & 9.03 & 4.73 & 8.46 &.. & .. & \\
\hline 21 & 21.96 &.. & 11.18 & 9.57 & 4.43 & ... &.. &. & \\
\hline 22 & 23.83 & .. & 10.15 & 10.67 & $4.4 !$ & .. & .. &. & \\
\hline 23 & 23.60 &.. & 9.64 &.. & ... & .. &.. &. & \\
\hline 24 & 23.36 &.. & 8.95 &.. &.. &.. & .. & .. & \\
\hline Average & 23.14 & 26.15 & 9.55 & 10.50 & 4.82 & 8.03 & 11.06 & 6.82 & 100.07 \\
\hline Std & 5.7874 & 6.9411 & 5.8507 & 4.5146 & 3.9549 & 2.8719 & 3.2325 & 2.3752 & \\
\hline a & 25.6161 & 28.510 & 13.016 & 12.770 & 7.306 & 9.405 & 12.243 & 7.789 & \\
\hline b & 20.1889 & 22.000 & 7.530 & 8.536 & 3.597 & 6.715 & 9.211 & 5.562 & \\
\hline R.I\% & 23 & 26 & 10 & 10 & 5 & 8 & 11 & 7 & 100 \\
\hline
\end{tabular}

لتحديد درجة التطابق لقيمة كل معيار من المعايير الثمانية تم اعتماد فترة ثقة (Confidence Interval 99\%).

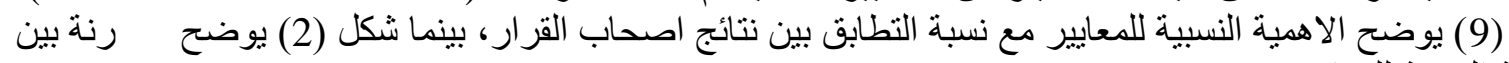
الاهية النسبية للمعايير.

(9) نسبة الاهمية للمعايير مع نسبة التطابق المبيق

\begin{tabular}{|c|c|c|c|c|c|c|c|c|}
\hline المسارات الخطية & & الوديان & البيئتي & طبيعة & الميل & المائية & & \\
\hline $7 \%$ & $11 \%$ & $8 \%$ & $5 \%$ & $10 \%$ & $10 \%$ & $26 \%$ & $23 \%$ & \\
\hline 16 & 19 & 20 & 22 & 22 & 24 & 15 & 24 & عدد المطابقين من \\
\hline $47 \%$ & $56 \%$ & $59 \%$ & $65 \%$ & $65 \%$ & $70 \%$ & $44 \%$ & $70 \%$ & \\
\hline
\end{tabular}




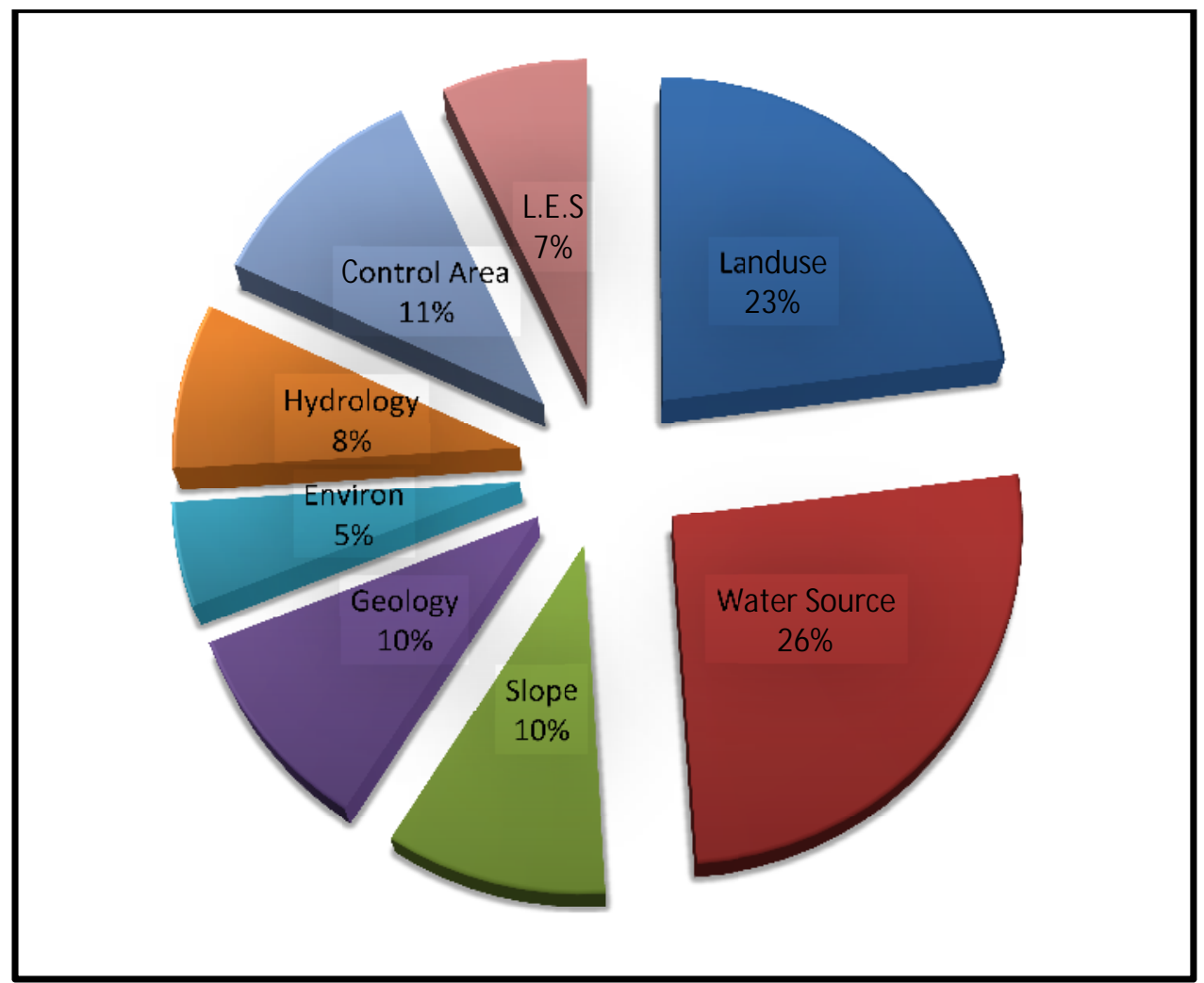

(2) المقارنة بين الاهمية النسبية للمعايير

\section{تحليد مسار الطريق:}

لتحديد مسار الطريق يتم توظيف البيانات التي تحديدها للمعايير المكانية لتحويلها الى خر ائط رقمية وتكوين قاعدة بيانات ضمن مقياس موحد لتحقيق عملية التركيب الخطي الموزون لاستخدامها في عملية التحليل باستخدام ضمن برنامج نظم المعلومات الجغر افية.

\section{والتوجيهات}

من خلال هذه الدر اسة واجر اء عملية التحليل لآر اء صانعي القرار وتحليل المعايير لتحديد مسار الطريق، نم التوصل الى مجمو عة من الاستنتاجات وكما يلي:

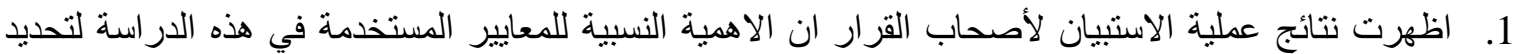

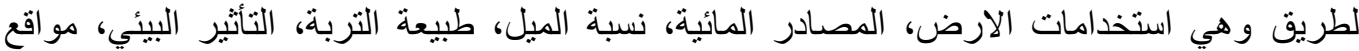

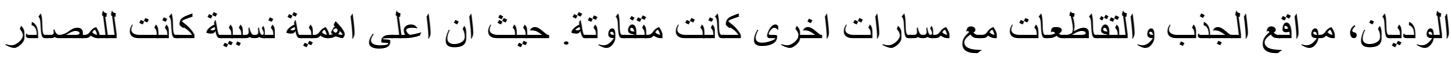

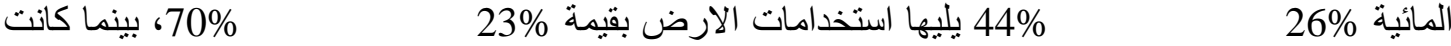

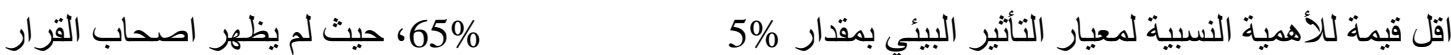

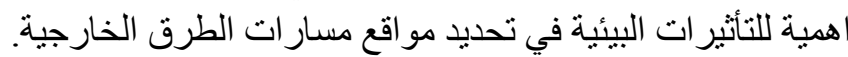

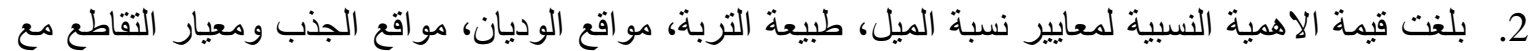
المسار ات الاخرى هي 10\% 


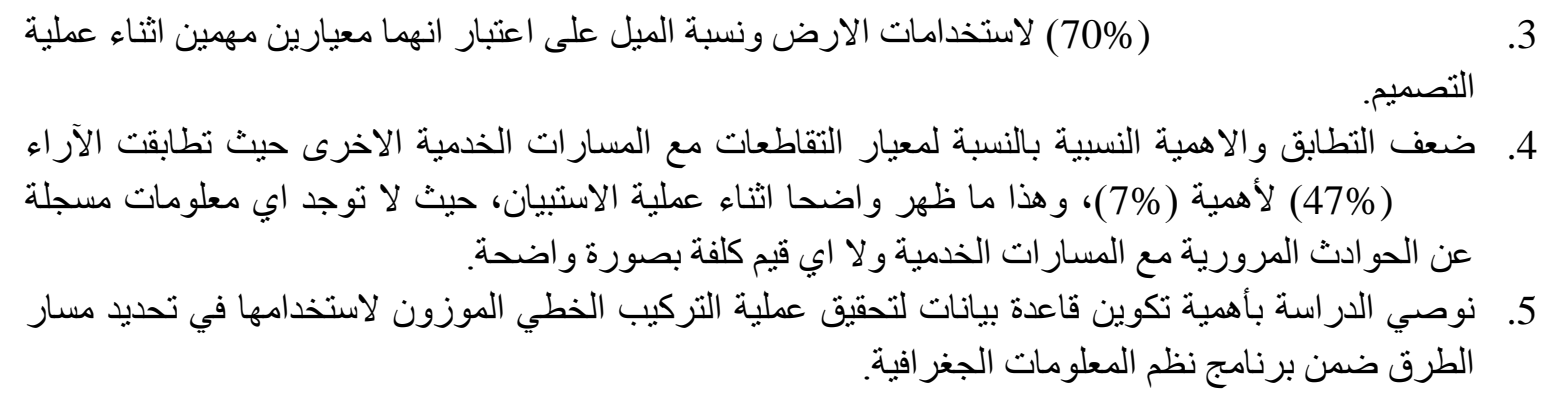

References:

1. Randal Greene, Rodolphe Devillers, Joan E. Luther and Brian G. Eddy(2011)." GISBased Multiple-Criteria Decision Analysis" Department of Geography, Memorial University of Newfoundland, Geography Compass 5/6 (2011): 412-432.

2. Saaty, T.L. ( 1980)." The Analytic Hierarchy Process", McGraw-Hill, New York, NY.

3. Saaty L. Thomas (2008)." Decision making with the analytic hierarchy process" University of Pittsburgh, Int. J. Services Sciences, Vol. 1, No. 1,2008.

4. Malczewski, J. (1999). "GIS and Multi-Criteria Decision Analysis". New York: J. Wiley \& Sons.

5. Mongkut \& Nattapon (2003). "Evaluation of Alternatives in Transportation Planning using Multi-Stakeholders Multi-Objectives AHP Modeling" School of Building Facilities and Civil Engineering, Sirindhorn International Institute of Technology Thammasat University, Proceedings of the Eastern Asia Society for Transportation Studies, Vol.4, October, 2003.

6. Djenaliev A.(2007)" Multi-criteria Decision Making and GIS for Railroad planning in Kyrgyzstan" requirement for the degree of Master, School of Architecture and the Built Environment Royal Institute of Technology Stockholm, Sweden.

7. Drobne S. and Lisec A.(2009)." Multi-attribute Decision Analysis in GIS: Weighted Linear Combination and Ordered Weighted Averaging" University of Ljubljana, Faculty of Civil and Geodetic Engineering, Jamova 2, SI-Ljubljana, Slovenia.

8. Mann H. Stuart.(1995) " Using The Analytic Hierarchy Process for Decision Making in Engineering Application :Some Challenges" Department of Industrial and Manufacturing Systems Engineering Louisiana State University, (C) International Journal of Industrial Engineering ISSN 1072-4761, U.S.A.

9. Sadasivuni R., O’Hara G. Charles, Nobrega R.\& Dumas J.(2009) "A Transportation Corridor Case study for Multi-Criteria Decision Analysis". Geo-systems Research Institute, Mississippi State University. ASPRS Annual Conference Baltimore, Maryland March 9-13.

$$
\text { تم اجراء البحث في كلية الهندسة = }
$$

